\title{
Photogenerated Electrical Fields for Biomedical Applications
}

\author{
Giuseppina Polino ${ }^{\dagger}$, Claudia Lubrano ${ }^{\dagger}$, Giuseppe Ciccone ${ }^{\dagger}$ and Francesca Santoro* \\ Center for Advanced Biomaterials for Healthcare, Istituto Italiano di Tecnologia, Naples, Italy
}

\section{OPEN ACCESS}

Edited by:

Gianni Ciofani,

Politecnico di Torino, Italy

Reviewed by:

Ilaria Elena Palamà,

Istituto di Nanotecnologia

(NANOTEC), Italy

Erhan Zor,

Necmettin Erbakan University, Turkey

*Correspondence:

Francesca Santoro

francesca.santoro@iit.it

tThese authors have contributed equally to this work

Specialty section:

This article was submitted to Nanobiotechnology,

a section of the journal Frontiers in Bioengineering and

Biotechnology

Received: 23 September 2018 Accepted: 23 October 2018

Published: 09 November 2018

Citation:

Polino G, Lubrano C, Ciccone G and

Santoro F (2018) Photogenerated

Electrical Fields for Biomedical

Applications.

Front. Bioeng. Biotechnol. 6:167.

doi: 10.3389/fbioe.2018.00167
The application of electrical engineering principles to biology represents the main issue of bioelectronics, focusing on interfacing of electronics with biological systems. In particular, it includes many applications that take advantage of the peculiar optoelectronic and mechanical properties of organic or inorganic semiconductors, from sensing of biomolecules to functional substrates for cellular growth. Among these, technologies for interacting with bioelectrical signals in living systems exploiting the electrical field of biomedical devices have attracted considerable attention. In this review, we present an overview of principal applications of phototransduction for the stimulation of electrogenic and non-electrogenic cells focusing on photovoltaic-based platforms.

Keywords: bioelectronics, photovoltaics, tissue engineering, biointerfaces, electrical stimulation

\section{INTRODUCTION}

Bioelectronics devices are meant to probe and stimulate biological entities through electricity by adopting smart biocompatible materials which are also conductive (Liao et al., 2015). In particular, traditional inorganic conductors, semiconductors and, more recently introduced, conjugated polymers had find major application in the development of bioelectronic platforms (Zhang and Lieber, 2016).

Electrical fields can be generated either intrinsically or upon transduction as, for instance, in photovoltaic materials, where light can induce the generation of relevant currents through the bulk of a photoconductive material.The materials in which this phenomenon happens can be inorganic, fully organic, or hybrid mixtures.

In particular, electrical fields can be exploited to locally stimulate cells and tissue inducing responses of different nature, for instance, altering the electrophysiological activity of electrogenic cells or modulate certain cellular processes and functionalities, i.e., polarity, proliferation, differentiation, in non-electrogenic cells (Blau, 2013; Pennacchio et al., 2018).

For example, silicon-based devices are widely employed for electrical interfaces both at the micro and nanometer scale with neural tissues (Thukral et al., 2018) and, furthermore, have gained major interest in the niche of photovoltaic-based platforms for retinal implants (Di Maria et al., 2018). However, these materials exhibit some limitations in terms of flexibility and stiffness in general, which make the cell-device coupling still not optimal.

In the last decade, new generation of hybrid or fully organic, highly biocompatible and functionally self-powered prostheses have been developed to treat, among various applications, blindness proposing a cutting-edge paradigm to interface phototransductive materials with biological cells (Khraiche et al., 2013; Maya-Vetencourt et al., 2017; Benfenati and Lanzani, 2018; Ferlauto et al., 2018). In addition, photovoltaic platforms have been recently proposed to directly interface non-electrogenic cells, such as fibroblasts, to trigger their proliferation and open up the possibility to use such materials as optimal candidate for wound healing purposes (Jin et al., 2013). 
Here, we present an overview of the main applications photovoltaic-based platforms have found in the recent years to mimic biological component with similar basic functionalities, i.e., retina, and in addition we aim to highlight the important potential of those materials have for cell stimulation in general.

\section{ELECTRICAL PHOTOTRANSDUCTION IN DEVICES FOR BIOMEDICAL APPLICATIONS}

Electrical phototransduction is the process through which photons are converted into electrical signals. For instance, inorganic semiconductors are exploited for their capacity to convert light into electrons flow by means of their P-N junction (Richter et al., 2018). However, their rigid nature, together with their poor biocompatibility has generated a major attention toward their organic counterparts for biomedical applications. Organic photovoltaic devices are able to deliver photocurrents thanks to the generation of electrical fields when light is absorbed in their photoactive layers, composed of a donor, and an acceptor semiconducting material. The donor material donates electrons transporting holes while the acceptor material withdraws electrons and further transports them (Polino et al., 2015).

In both inorganic and organic cases, charges are finally transported to an electrode, which can be in contact with a biological cell or a tissue. Starting from the carrier transport at the electrode, an electrical field is generated between the device and the biological matter and this bioelectronic coupling can modulate biological processes at different matter of scale and cellular architectures (Pennacchio et al., 2018).

The effects of this interface on cells can be investigated by observing capacitive, chemical and thermal mechanisms involving the cell membrane (Di Maria et al., 2018). In particular, platforms based on electrical photo-transduction have aroused the interest of many research groups with the purposes of recording and stimulating single cells, cell networks or tissues (Sim et al., 2014; Chenais et al., 2017; Jeong et al., 2017).

For example, in the work of Ghezzi et al. (2011), first attempts have successfully shown that stimulation of primary neurons via light absorption in a P3HT-based biointerface is possible. In particular, the bioelectronic interface consisted in a very thin film $(\approx 150 \mathrm{~nm})$ of a P3HT:PCBM blend deposited on an ITO-coated glass substrate. One step further was accomplished by the same group using a platform for neuronal stimulation which consisted of an active layer only in the n-type conjugated polymer P3HT (Ghezzi et al., 2013). This is just an example of how photo-transduction can take place in $2 \mathrm{D}$ materials and how this phenomenon can be exploited for generating electrical fields to trigger certain processes at the membrane of cells. However, it is interesting to note that even bioelectronic platforms are moving fast toward more biomimetic approaches inspired by those of tissue engineering (Pennacchio et al., 2018).

In this context, first attempts to create $3 \mathrm{D}$ photovoltaic platforms for skin regeneration have been carried and the local photo-transduction mechanism of $3 \mathrm{D}$ meshes is still under discussion (Jin et al., 2014). Here, we explore the use of light photo-trasduction effect on single cells particularly focusing on tuning cellular behavior and act as cell-instructive platforms for various applications such as platforms for immunorecognition, or as activation/inhibition of the activity of electrogenic and non-electrogenic cells.

\section{ELECTROGENIC CELLS}

Platforms based on transduction of photons in to current/voltage generation have found major applications in the modulation of the electrical activity in electrogenic cells. In fact, these cells, such as cardiomyocytes or neuronal cells, are capable of changing their membrane potential upon stimuli generating fast-changing events (action potentials). Thus, using certain electrical fields can be a suitable approach to trigger action potential inhibition or activation (Love et al., 2018).

Moreover, photovoltaic platforms has found a niche in the design of implantable devices for restoring lost functionalities in the retina (Benfenati and Lanzani, 2018; Di Maria et al., 2018).

Numata and co-workers reported the first example of photoinhibition of ion transport in PC-12 cells by using chargeseparation molecules ferrocene $(\mathrm{Fc})$-porphyrin $(\mathrm{P})-\mathrm{C} 60$ linked triads. This compound was delivered close to the plasma membrane using drug carriers and, after light stimulation a depolarization of the membrane potential and an inhibition of potassium channels was observed. This result suggests thatmore sophisticated molecules can lead to the control of firingneuronal cells (Numata et al., 2012).

Abdullaeva and co-workers, with the intent to create organicbased artificial photoreceptors, developed a photoactive layer consisting of an anilino-squaraine donor blended with a fullerene acceptor as support for N2A cells (neuronal model cell line) growth. They supposed that during the pulse stimulation there is an accumulation of negative charge carriers at the photoconductor-electrolyte interface (Figure 1A), which results into cell depolarization. When the light is turned off instead, a rapid hyperpolarization of the cell membrane was detected (Abdullaeva et al., 2016). Similarly, induced photocapacitance can be exploited for modulation of the membrane potential in cells.

In fact, Martino and co-workers observed a similar effect on human embryonic kidney (HEK-293) cells growth on a conjugated polymer poly(3-hexylthiophene) (P3HT). The local heating of the material produced an increase in the ion transport through membrane channels, causing a decrease of the membrane resistance (Martino et al., 2015). Recently, Glowacki and co-workers have proposed an approach for neural photostimulation employing an electrolytic photocapacitor (Figure 1B) built with a trilayer of metal and $\mathrm{p}-\mathrm{n}$ semiconducting organic nanocrystals (Rand et al., 2018).

Moving toward the coupling of photovoltaic platforms with tissues, retina implants have found major interest in the last years (Benfenati and Lanzani, 2018). Photovoltaic devices which are foldable and flexible have been developed for wide-field epiretinal 


\section{Abdullaeva et. al.2016}

A

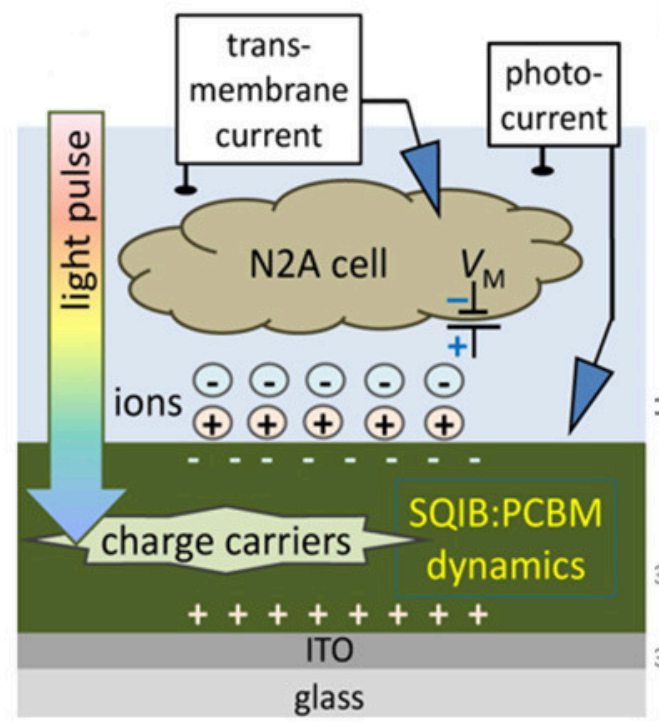

Ferlauto et. al. 2018

\section{Rand et. al. 2018}

B
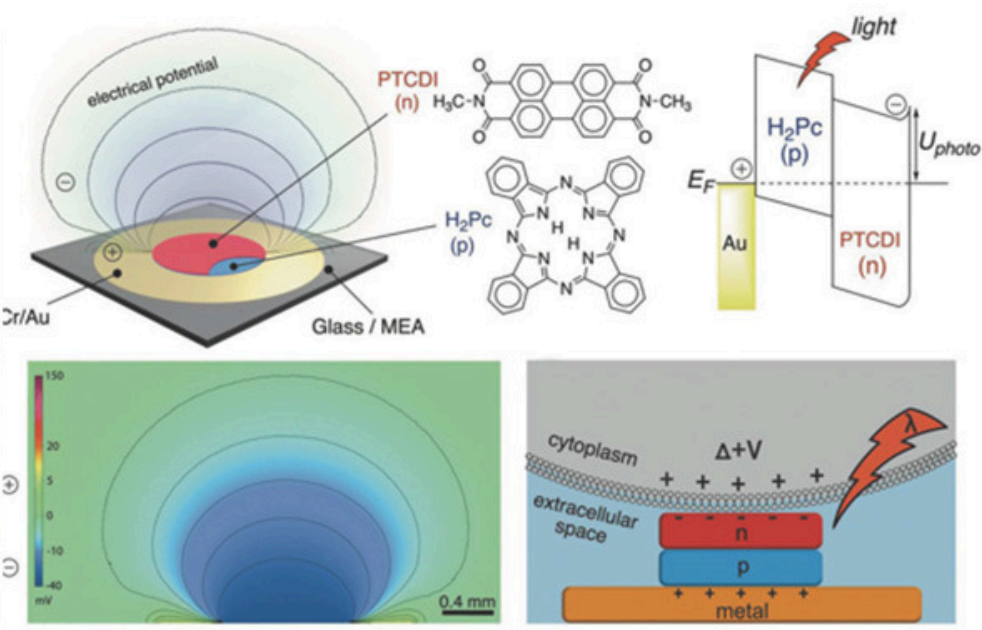

Flores et. al.2018

C

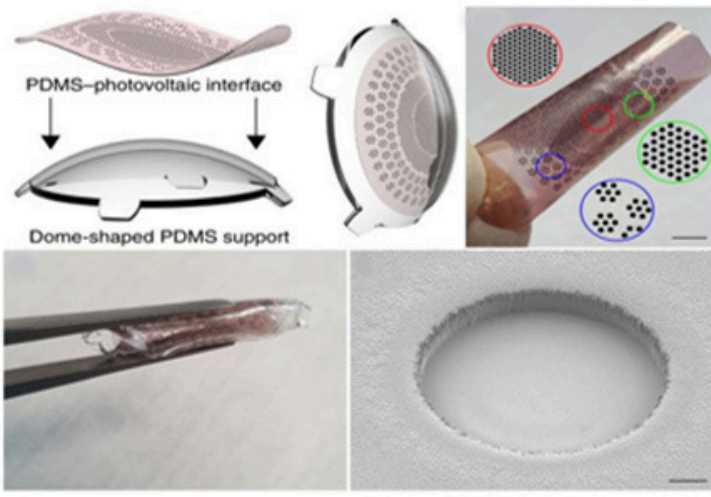

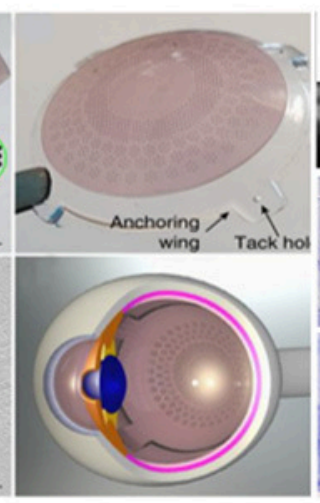

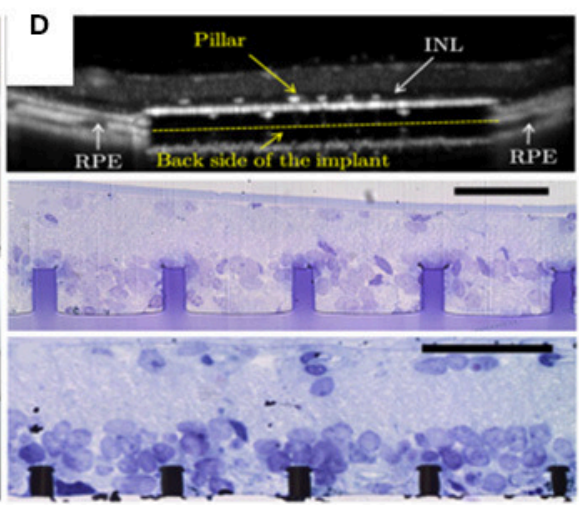

FIGURE 1 | (A) Schematic of experimental setup for transient photocurrent generation through a fullerene film. Reprinted with permission from Abdullaeva et al. (2016). (B) Layout of the photocapacitor, molecular structures of the pigment semiconductors and energy band illustration of a metal-p-n device. Reprinted with permission from Rand et al. (2018). (C) 3D photovoltaic wide-field retinal prosthetics realized on a PDMS support (POLYRETINA) Reprinted with permission from Ferlauto et al. (2018). (D) Pillar-based subretinal implant. Reprinted with permission from Flores et al. (2018).

prosthesis. These devices are capable of stimulating wireless retinal ganglion cells. The material stack included poly $(3,4-$ ethylenedioxythiophene) polystyrene sulfonate (PEDOT:PSS) as anode and Poly(3-hexylthiophene) (P3HT) blend based as photoactive layer (Figure 1C) (Maya-Vetencourt et al., 2017; Ferlauto et al., 2018).

Retinal and subretinal inorganic prostheses were developed to restore sight in patients blinded by retinal degeneration by stimulating the inner retinal neurons using $3 \mathrm{D}$ pillar electrodes (Figure 1D) which enhance the cell-chip coupling and the integration the implant in the target tissue (Mathieson et al., 2012; Flores et al., 2018). In a recent work done by Ho et al. (2018a) a photodiode array made of boron-doped silicon-oninsulator (SOI) wafers was used to perform in vivo and in vitro measurements, in which the retina is placed between the recording array (ganglion cell side) and the photodiode array (photoreceptor side). In a recent work, transparent extracellular microelectrode arrays (MEA) were used to characterize the spatial and temporal response properties of retinal ganglion cells (RGCs) to photovoltaic stimulation in the healthy and degenerate rat retina (Ho et al., 2018b). It was demonstrated that using silicon photodiodes to build photovoltaic pixels arrays and it is possible to convert signals into patterns of current to stimulate the inner retinal neurons for wireless 
neural stimulation in translucent tissues (Boinagrov et al., 2016).

\section{NON-ELECTROGENIC CELLS}

Beside electrogenic cells and tissues, photoelectrical devices can be used also for promoting cellular proliferation, for instance, in tissue regeneration applications.

One successful approach in tissue engineering is the use of photovoltaic platforms as novel treatments for angiogenesis. In this approach, a solar cell generates an electrical field promoting the formation of capillaries and arterioles at the ischemic region, attenuating muscle necrosis and fibrosis and promoting the secretion of angiogenic growth factors and the migration of mesenchymal stem cells (MSCs), myoblasts, endothelial progenitor cells, and endothelial cells in in vitro experiments (Figure 2A) (Jeong et al., 2017).

Many efforts are carried out on different types of cells like adipose-derived stem cells (ASC) and endothelial cells, evaluating the influence of pulsed LED light of three different wavelengths using continuous LLLT stimulation (Rohringer et al., 2017). In Couto et al., it was shown that a treatment of skin lesions with coherent and incoherent light sources (laser and LED, respectively) enables the wound healing process in elderly rats, with good results in terms of collagen deposition, fibroblasts proliferation and inflammatory cellular response (Couto et al., 2017). In fact, other studies have been developed on fibroblasts treatment using laser or led therapy to inhibit keloid fibroblasts after irradiation (Couto et al., 2017; Lee et al., 2017). In the end, different studies demonstrated the importance of therapy with coherent light in the visible spectrum to optimize the process of tissue repair, and some studies suggests that comparable effectiveness could be reached with the lower costs non-coherent light in the same spectral region.

Another interesting approach involved the fabrication of nanoweb substrates made of poly(3-hexylthiophene) (P3HT) to enhance the neurogenesis of human fetal neural stem cells (hfNSCs) and control their behavior via optoelectrical stimulation (Figure 2B) (Yang et al., 2017). Low-level laser therapy (LLLT) based on low-level laser or lightemitting diodes (LEDs) was used for tissue regeneration in mouse neural stem cell on 3D printed scaffolds (Zhu et al., 2017).

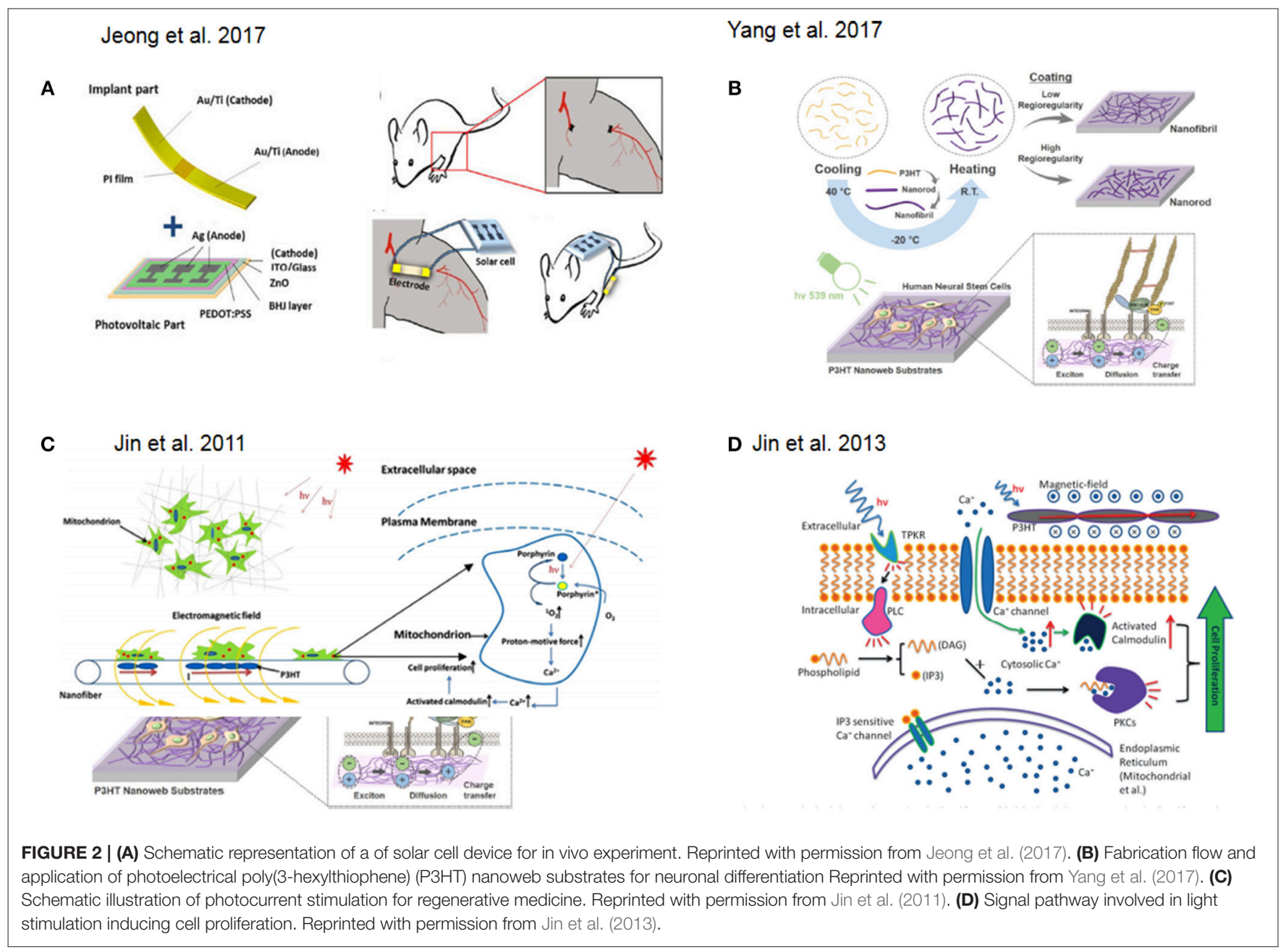


An interesting novelty regards the possibility to treat diseases delivering cellsto an injured or diseased organ/tissue. Usually this is accomplished by collecting cells through a digestion process with enzymes which can lead to Undesired enzymatic residuces. In orer to overcome this, cells have been cultured onsilicon based photovoltaic surfaces have been released through light stimulation as presented in the work of Bhuyan et al. (2016).

In a recent work done by Diring et al. (2017), photoactive zirconium-based metal organic framework (MOF) particles embedded in a polymer matrix were employed as growth substrate for HeLa cells. The substrate was able to release CO upon light irradiation, and its subsequent cellular uptake was monitored using a fluorescent probe.

In this context, one example, which also attempted to recapitulate $3 \mathrm{D}$ tissue-like environment with particular focus on regenerative medicine, was presented by Jin and co-workers. They reported on the photocurrent stimulation effect on cells with particular focus on understanding the combined effect of direct light interaction together with the responseto the induced electromagnetic field. In fact, porphyrins are activated by photons interactions while calcium ions translocate through the voltagegated channels at the membrane due to the electromagnetic field. Overall, this results in an increase of cytosolic $\mathrm{Ca}^{2+}$ which might trigger protein pathways responsible for cell proliferation (Jin et al., 2011) (Figure 2C). Furthermore, they showed how to combine $3 \mathrm{D}$ structures with photoactive materials in order to achieve skin regeneration. In particular, they used human dermal fibroblasts (HDFs) and prepared a photosensitive nanofibrous scaffolds by electrospinning using Poly(3-hexylthiophene) (P3HT) and Polycaprolactone (PCL) as base materials to fabricate the nanofibers. Here, they further discussed how the cytosolic $\mathrm{Ca} 2+$ increase would led to the activation of the low molecular weight protein calmodulin, which causes the activation of several key intracellular processes leading to cell division (Tomlinson et al., 1984) in combination with direct light activation of protein kinase C. As a result, these two effects led to an increase in cell proliferation (Jin et al., 2013, 2014) (Figure 2D). Furthermore, Jin and co-workers designed a new photosensitive semiconductive polymer, PDBTT (poly (N,N-bis

\section{REFERENCES}

Abdullaeva, O. S., Schulz, M., Balzer, F., Parisi, J., Lützen, A., Dedek, K., et al. (2016). Photoelectrical stimulation of neuronal cells by an organic semiconductor-electrolyte interface. Langmuir 32, 8533-8542. doi: 10.1021/acs.langmuir.6b02085

Benfenati, F., and Lanzani, G. (2018). New technologies for developing second generation retinal prostheses. Lab Anim. 47, 71-75. doi: 10.1038/s41684-018-0003-1

Bhuyan, M. K., Rodriguez-Devora, J., Tseng, T.-L. B., and Boland, T. (2016). Photovoltaic surfaces enable clonal myoblastic cell release using visible light as external stimulation. Biotechnol. J. 11,393-398. doi: 10.1002/biot.201500126

Blau, A. (2013). Cell adhesion promotion strategies for signal transduction enhancement in microelectrode array in vitro electrophysiology: an introductory overview and critical discussion. Curr. Opin. Colloid Interface Sci. 18, 481-492. doi: 10.1016/j.cocis.2013.07.005

Boinagrov, D., Lei, X., Goetz, G., Kamins, T. I., Mathieson, K., Galambos, L., et al. (2016). Photovoltaic pixels for neural stimulation: circuit (2-octyldodecyl)-3,6-di(thiophen-2-yl)-2,5-dihydropyrrolo[3,4c]pyrrole- 1,4-dione-alt-thieno[3,2-b]thiophene), with maximum absorbance at $600 \mathrm{~nm}$. This polymer with nanofibers of PCL incorporated, was electrospun and tested to study the proliferative effect of PCL/PDBTT nanofibers on HDFs under red LED illumination (Jin et al., 2017). Another interesting study showed how a scaffold made of electrospun fiber of $\mathrm{P} 3 \mathrm{HT}$ in combination with a typical polymer used for tissue engineering, poly(L-lactic acid)-co-poly-(e-caprolactone) could be successfully used as photosensitive platforms for enhancing fibroblast proliferation and thus as good precursor of a wound dressing solution (Jin et al., 2014).

\section{CONCLUSION}

In this review, we reported the last advances in electrical phototransduction applied to biomedical applications. In particular, we focused the attention on the possible effects of photoelectrical stimulation driven on cells and tissues. The aim was to report the behavior of the biological system at the biotic-abiotic interface considering various approaches for enhancement/inhibition of electrogenic and non-electrogenic cells functionalities. In particular, we highlighted how the use of photovoltaic platforms had found major application in designing retinal prosthetics. Moreover, organic photovoltaic materials are used for building new bio-compliant platforms and, in perspective, represent the foremost class of material for cell-chip coupling. We foresee an increasing application of these materials also for tissue engineering purposes as preliminarily proposed for wound healing. Furthermore, the few examples of photovoltaic-based scaffolds paved the way to advanced platforms which need to be developed toward more biomimetic $3 \mathrm{D}$ environments.

\section{AUTHOR CONTRIBUTIONS}

All authors listed have made a substantial, direct and intellectual contribution to the work, and approved it for publication. models and performance. IEEE Trans. Biomed. Circuits Syst. 10, 85-97. doi: 10.1109/TBCAS.2014.2376528

Chenais, N., Leccardi, A., Ildelfonsa, M. J., Ferlauto, L., Sivula, K., and Ghezzi, D. (2017). Photovoltaic Stimulation of Retinal Ganglion Cells with Wide-Field Epiretinal Prosthesis. Available online at: https://infoscience.epfl.ch/record/ 231326 (Accessed August 30, 2018).

Couto, J. P. A., do, Nicolau, R. A., Munin, E., Castillo, M. A. S., Fadin, -Priscila Silva, L., et al. (2017). Skin tissue healing induced by coherent (laser) or noncoherent (led) light therapy on aged rats. Int. Phys. Med. Rehabil. J. 1, 1-6. doi: 10.15406/ipmrj.2017.1.00029

Di Maria, F., Lodola, F., Zucchetti, E., Benfenati, F., and Lanzani, G. (2018). The evolution of artificial light actuators in living systems: from planar to nanostructured interfaces. Chem. Soc. Rev. 47, 4757-4780. doi: 10.1039/C7CS00860K

Diring, S., Carné-Sánchez, A., Zhang, J., Ikemura, S., Kim, C., Inaba, H., et al. (2017). Light responsive metal-organic frameworks as controllable CO-releasing cell culture substrates. Chem. Sci. 8, 2381-2386. doi: $10.1039 /$ c6sc04824b 
Ferlauto, L., Leccardi, M. J. I. A., Chenais, N. A. L., Gilliéron, S. C. A., Vagni, P., Bevilacqua, M., et al. (2018). Design and validation of a foldable and photovoltaic wide-field epiretinal prosthesis. Nat. Commun. 9:992. doi: 10.1038/s41467-018-03386-7

Flores, T., Lei, X., Huang, T., Lorach, H., Dalal, R., Galambos, L., et al. (2018). Optimization of pillar electrodes in subretinal prosthesis for enhanced proximity to target neurons. J. Neural Eng. 15:036011. doi: 10.1088/1741-2552/aaac39

Ghezzi, D., Antognazza, M. R., Maccarone, R., Bellani, S., Lanzarini, E., Martino, N., et al. (2013). A polymer optoelectronic interface restores light sensitivity in blind rat retinas. Nat. Photonics 7, 400-406. doi: 10.1038/nphoton.2013.34

Ghezzi, D., Antognazza, M. R., Maschio, M. D., Lanzarini, E., Benfenati, F., and Lanzani, G. (2011). A hybrid bioorganic interface for neuronal photoactivation. Nat. Commun. 2:166. doi: 10.1038/ncomms1164

Ho, E., Lorach, H., Goetz, G., Laszlo, F., Lei, X., Kamins, T., et al. (2018a). Temporal structure in spiking patterns of ganglion cells defines perceptual thresholds in rodents with subretinal prosthesis. Sci. Rep. 8:3145. doi: 10.1038/s41598-018-21447-1

Ho, E., Smith, R., Goetz, G., Lei, X., Galambos, L., Kamins, T. I., et al. (2018b). Spatiotemporal characteristics of retinal response to networkmediated photovoltaic stimulation. J. Neurophysiol. 119, 389-400. doi: 10.1152/jn.00872.2016

Jeong, G.-J., Oh, J. Y., Kim, Y.-J., Bhang, S. H., Jang, H.-K., Han, J., et al. (2017). Therapeutic Angiogenesis via Solar Cell-Facilitated Electrical Stimulation. ACS Appl. Mater. Interfaces 9, 38344-38355. doi: 10.1021/acsami.7b13322

Jin, G., Li, J., and Li, K. (2017). Photosensitive semiconducting polymerincorporated nanofibers for promoting the regeneration of skin wound. Mater. Sci. Eng. C 70, 1176-1181. doi: 10.1016/j.msec.2016.04.107

Jin, G., Prabhakaran, M. P., Kai, D., Kotaki, M., and Ramakrishna, S. (2013). Electrospun photosensitive nanofibers: potential for photocurrent therapy in skin regeneration. Photochem. Photobiol. Sci. 12, 124-134. doi: $10.1039 / \mathrm{c} 2 \mathrm{pp} 25070 \mathrm{e}$

Jin, G., Prabhakaran, M. P., Liao, S., and Ramakrishna, S. (2011). Photosensitive materials and potential of photocurrent mediated tissue regeneration. J. Photochem. Photobiol. B 102, 93-101. doi: 10.1016/j.jphotobiol.2010.09.010

Jin, G., Prabhakaran, M. P., and Ramakrishna, S. (2014). Photosensitive and biomimetic core-shell nanofibrous scaffolds as wound dressing. Photochem. Photobiol. 90, 673-681. doi: 10.1111/php.12238

Khraiche, M. L., El Emam, S., Akinin, A., Cauwenberghs, G., Freeman, W., and Silva, G. A. (2013). Visual evoked potential characterization of rabbit animal model for retinal prosthesis research. Conf. Proc. IEEE Eng. Med. Biol. Soc. 2013, 3539-3542. doi: 10.1109/EMBC.2013.6610306

Lee, H. S., Jung, S.-E., Kim, S. K., Kim, Y.-S., Sohn, S., and Kim, Y. C. (2017). Low-level light therapy with $410 \mathrm{~nm}$ light emitting diode suppresses collagen synthesis in human keloid fibroblasts: an in vitro study. Ann. Dermatol. 29, 149-155. doi: 10.5021/ad.2017.29.2.149

Liao, C., Zhang, M., Yao, M. Y., Hua, T., Li, L., and Yan, F. (2015). Flexible organic electronics in biology: materials and devices. Adv. Mater. 27, 7493-7527. doi: 10.1002/adma.201402625

Love, M. R., Palee, S., Chattipakorn, S. C., and Chattipakorn, N. (2018). Effects of electrical stimulation on cell proliferation and apoptosis. J. Cell. Physiol. 233, 1860-1876. doi: 10.1002/jcp.25975

Martino, N., Feyen, P., Porro, M., Bossio, C., Zucchetti, E., Ghezzi, D., et al. (2015). Photothermal cellular stimulation in functional bio-polymer interfaces. Sci. Rep. 5:8911. doi: 10.1038/srep08911

Mathieson, K., Loudin, J., Goetz, G., Huie, P., Wang, L., Kamins, T. I., et al. (2012). Photovoltaic retinal prosthesis with high pixel density. Nat. Photonics 6, 391-397. doi: 10.1038/nphoton.2012.104
Maya-Vetencourt, J. F., Ghezzi, D., Antognazza, M. R., Colombo, E., Mete, M., Feyen, P., et al. (2017). A fully organic retinal prosthesis restores vision in a rat model of degenerative blindness. Nat. Mater. 16, 681-689. doi: $10.1038 /$ nmat 4874

Numata, T., Murakami, T., Kawashima, F., Morone, N., Heuser, J. E., Takano, Y., et al. (2012). Utilization of photoinduced charge-separated state of donoracceptor-linked molecules for regulation of cell membrane potential and ion transport. J. Am. Chem. Soc. 134, 6092-6095. doi: 10.1021/ja3007275

Pennacchio, F. A., Garma, L. D., Matino, L., and Santoro, F. (2018). Bioelectronics goes 3D: new trends in cell-chip interface engineering. J. Mater. Chem. B. doi: 10.1039/C8TB01737A. [Epub ahead of print].

Polino, G., Casaluci, S., Dianetti, M., Dell'Elce, S., Liscio, A., Mirruzzo, V., et al. (2015). Inverted bulk-heterojunction solar cells using polyethylenimineethoxylated processed from a fully aqueous dispersion as electron-transport layer. Energy Technol. 3, 1152-1158. doi: 10.1002/ente.201500154

Rand, D., Jakešová, M., Lubin, G., Vebraite, I., David-Pur, M., Đerek, V., et al. (2018). Direct electrical neurostimulation with organic pigment photocapacitors. Adv. Mater. 30:1707292. doi: 10.1002/adma.201 707292

Richter, A., Benick, J., Fell, A., Hermle, M., and Glunz, S. W. (2018). Impact of bulk impurity contamination on the performance of high-efficiency ntype silicon solar cells. Prog. Photovolt. Res. Appl. 26, 342-350. doi: 10.1002/ pip. 2990

Rohringer, S., Holnthoner, W., Chaudary, S., Slezak, P., Priglinger, E., Strassl, M., et al. (2017). The impact of wavelengths of LED light-therapy on endothelial cells. Sci. Rep. 7:10700. doi: 10.1038/s41598-017-11061-y

Sim, S. L., Szalewski, R. J., Johnson, L. J., Akah, L. E., Shoemaker, L. E., Thoreson, W. B., et al. (2014). Simultaneous recording of mouse retinal ganglion cells during epiretinal or subretinal stimulation. Vision Res. 101, 41-50. doi: 10.1016/j.visres.2014.05.005

Thukral, A., Ershad, F., Enan, N., Rao, Z., and Yu, C. (2018). Soft ultrathin silicon electronics for soft neural interfaces: a review of recent advances of soft neural interfaces based on ultrathin silicon. IEEE Nanotechnol. Mag. 12, 21-34. doi: 10.1109/MNANO.2017.2781290

Tomlinson, S., MacNeil, S., Walker, S. W., Ollis, C. A., Merritt, J. E., and Brown, B. L. (1984). Calmodulin and cell function. Clin. Sci. Lond. Engl. 66, 497-507.

Yang, K., Oh, J. Y., Lee, J. S., Jin, Y., Chang, G.-E., Chae, S. S., et al. (2017) Photoactive poly(3-hexylthiophene) nanoweb for optoelectrical stimulation to enhance neurogenesis of human stem cells. Theranostics 7, 4591-4604. doi: 10.7150/thno.20169

Zhang, A., and Lieber, C. M. (2016). Nano-Bioelectronics. Chem. Rev. 116, 215-257. doi: 10.1021/acs.chemrev.5b00608

Zhu, W., George, J. K., Sorger, V. J., and Zhang, L. G. (2017). 3D printing scaffold coupled with low level light therapy for neural tissue regeneration. Biofabrication 9:025002. doi: 10.1088/1758-5090/aa6999

Conflict of Interest Statement: The authors declare that the research was conducted in the absence of any commercial or financial relationships that could be construed as a potential conflict of interest.

Copyright (c) 2018 Polino, Lubrano, Ciccone and Santoro. This is an open-access article distributed under the terms of the Creative Commons Attribution License (CC $B Y)$. The use, distribution or reproduction in other forums is permitted, provided the original author(s) and the copyright owner(s) are credited and that the original publication in this journal is cited, in accordance with accepted academic practice. No use, distribution or reproduction is permitted which does not comply with these terms. 